plus ou moins prolixe qu'il faut écrire: ce sont des mceurs qu'il faut créer, c'est l'opinion qu'il faut éclairer, l'opinion de tout le monde, de vous, de moi, du voisin, du passant qui chemine sur la route... Notre société a la prétention d'ètre une démocratie, sa fol ce est donc dans ce que pense $M$. Tout le monde, elle réside dans l'opinion publique. Ia loi, en tout pays, latin ou germanique, ne vaut que par les mours. C'est vieux comme le monde, ce que je dis là!

Une autre vielllerie, qui a bien son prix aussi, est que, plus la loi est brève, mieux elle est obéie, respectée; que, plus elle est copieuse, plus elle offre d'occasions de distinctions subtiles permettant de la tourner.

L'abus! Certes oui je le condamne aussi énergiquement que quiconque; mais, outre qu'il est souvent difficile à discerner et à définir, il a une forme particulièrement redoutable et odieuse, c'est l'abus de pouroir. Les autres abus, avec de la vigilance et des juges, on en vient à bout sans trop de peine, mais non de lui. Tous ceux qui ont l'expérience de la vie pourraient apporter ici un important contingent d'exemples probants. C'est cet abus-là auquel il faut d'abord barrer la route: or, il vient à l'abri du texte législatif et toute loi nouvelle en introduit le germe, dont des administrateurs éclairés et des juges équitables ne peuvent pas toujours empêcher le développement. C'est pourquoi je compte plus sur la puissance des mours que sur celle de la lettre écrite.

Mais, les mours, il faut les créer, il faut endoctriner notre semblable, l'édifier, lui donner des raisons de se convaincre, de se faire une opinion personnelle. M. Bernard Brunhes acceptera certainement que, pour atteindre, en matière de reboisement et de santé de la montagne, le résultat réel que lui et nous désirons, j’aie plus de confiance dans la chaleur de ses convictions, son talent de parole et de plume que dans un texte de loi qu'on laissera sommeiller ou que d'habiles spécialistes sauront toujours violer, exploiter ou tourner, tout en ayant l'air de le respecter.

Pardonnez-moi cette longue lettre et veuillez agréer. . . . .

Commandant Audebrand, Ingéneur, ancien éleve de l'Ecole polytechnique.

\section{Essais comparatifs de Jaugeages}

Quelle méthode doit-on employer en hydraulique pour mesurer un débit? That is the question! Les hydrauliciens ne sont pas d'accord, je n'ai pas à le faire savoir ici. Les uns prétendent: l'emploi du déversoir fournit le moyen de beaucoup le plus exact pour évaluer un débit, et il est inutile d'attendre la précision d'aucun autre procédé; les autres disent: quand l'emploi du déversoir n'est pas possible, on peut recourir au tube piézométrique ou au moulinet, car maniés par des opérateurs habitués à s'entourer des précautions voulues, ces appareils donnent des résultats d'une approximation suffisante dans la plupart des cas de la pratique industrielle. Enfin, il est des hydrauliciens qui hésitent encore beaucoup sur la préférence à donner à la méthode susceptible de la moindre erreur dans les circonstantes délicates comme celle de la mesure du rendement d'un moteur hydraulique: les formules de déversoir sont nombreuses, disent-ils, laquelle est la meilleure ? N'en est1 pas dans le cas de donner des résultats moins approchés que la mise en ceuvre, dans les meilleures conditions, du moulinet de Woltmann par exemple, et si les deux procédés sont applicables à la même mesure, faut-il prendre le déversoir avec telle formule ou telle autre, ou employer le moulinet?

Combien est désirable linvention du procédé tant cherché qui, nous permettant d'opérer sans erreur dans les diverses circonstances où on pourrait lui comparer les méthodes actuelles, nous fixerait sur leur degré d'approximation; je ne parle pas d'un procédé qui soit applicable aux mesures en rivière comme aux jaugeages d'une conduite forcée, mais d'un procédé qui nous donnant, par exemple, la valeur absolue du débit d'un canal, nous permettrait de faire simultanément, sur ce même débit, des mesures par déversoir et au moyen du tube piézométrique et du moulinet. On pourrait n'employer le dit procédé, s'il doit comporter des complications de mise en ceuvre, que dans les seules circonstances où la précision est de rigueur et se contenter des méthodes aujourd'hui usitées dans les cas, assez fréquents, où l'on peut s'en tenir à des résultats approchés; alors on serait au moins fixé str le degré d'approximation de ces miéthodes usuelles.

Il est évident, par exemple, que l'exactitude au $1 / 10^{\circ}$ près est suffisante dans les jaugeages de cours d'eau et que les résultats fournis, soit par le tube piézométrique, soit par le moulinet de Woltmann, méritent crédit quand ils proviennent d'opérateurs expérimentés. C'est donc la méthode fondée sur l'indication des vitesses par ces appareils qu'il est tout naturel d'employer en raison de sa simplicité de mise en ceuvre, pour le tracé des courbes de débit de nos cours d'eau. Mais, quand il s'agit de mesurer le volume d'eau qui actionne une turbine, la précision désirable est de l'ordre du r/100. Quand, pour faire les mesures, on peut disposer un déversoir sur les canaux d'amenée ou de fuite, on a des chances, en opérant avec soin et en choisissant 'la bonne formule (on verra pourquoi ja souligne), d'approcher de ce degré de précision. Mais quand, ne pouvant pas placer de déversoir dans des conditions convenables, on doit recourir, par exemple, à l'emploi du moulinet, à quelle erreur s'expose-t-on par rapport au résultat qui serait donné par l'application de la formule du déversoir la mieux choisie?

On a bien imaginé, récemment, pour mesurer le débit des usines hydrauliques, des méthodes très ingénieuses et susceptibles d'une précision de l'ordre du 1/100\%. Nos lecteurs les connaissent, car nous avons décrit ici, en détail, les méthodes Ribourt, Parenty et Mesnager, Bellet. On se rappelle qu'elles ont pour principe, soit de supprimer l'intervention de coefficients empiriques dans leurs formules caractéristiques de juugeage, soit d'éviter le fameux coefficient d'erreur personnel qui a tant d'importance dans les méthodes précédentes, et cela en demandant à des appareils enregistreurs susceptibles d'une précision presque absolue, de remplacer mécaniquement les lettres par leurs valeurs dans les formules de jaugeages. Malheureusement, ces méthodes ne sont encore, étant donné les dispositions actuelles de nos usines, que d'un emploi restreint; l'appa- 
reillage spécial qu'exige leur mise en œuvre ne les rend applicables que dans des circonstances peu nombreuses. Mais il faut espérer que devant l'importance des indications fournies par des mesures exactes de débit, les industriels se décideront, par la suite, à faire le nécessaire pour rendre ces méthodes d'un emploi commode.

L'expert-hydraulicien appelé à faire un jaugeage dans une usine pourra toujours se servir, soit du déversoir, soit du moulinet de Woltmann. S'il en est qui ne sont pas embarassés dans le choix de la méthode lorsque les deux sont d'application possible, ou dans le choix des formules lorsqu'il a été décidé que l'on opère avec un déversoir, nous devons déclarer que nous ne sommes pas de ceux-là. Nous ne saurions dire a priori quel procédé donnera les résultats les plus approchés de l'exactitude et notre choix, dans chaque cas, n'est motivé que par des considérations touchant les probabilités de moindre erreur.

Notre avis est que l'on doit employer le déversoir toutes les fois qu'on a le choix entre les deux moyens. La raison en est simple: même en admettant l'égalité dans la valeur théorique des deux méthodes, le déversoir offre tout au moins l'avantage pratique de ne nécessiter qu'une seule mesure, tandis que l'emploi du moulinet, pour peu que le canal à jauger soit de dimensions un peu grandes, entraîne à un grand nombre de mesures; il en résulte que le coefficient d'erreur personnel intervient beaucoup plus avec le moulinet qu'avec le déversoir.

Plusieurs hydrauliciens ont émis des doutes sur l'exactitude des mesures faites avec un moulinet dans les cours d'eau non canalisés. M. Rateau a, en effet, montré que, lorsque un moulinet est immergé dans un cours d'eau où les vitesses des filets liquides sont variables suivant l'espace et suivant le temps, cet appareil conduit à une estimation trop grande de la vitesse moyenne, car il indique, non pas la moyenne des vitesses des filets liquides, mais la moyenne des carrés des vitesses, autrement dit, la quantité de mouvement moyenne.

Mais dans les cours d'eau canalisés, comme le sont les canaux de dérivation, d'amenée ou de fuite des usines hydrauliques modernes, le mouvement de l'eau est assez régulier, et l'on peut alors se demander si, dans ce cas, les résultats auxquels conduit l'emploi d'un moulinet ne sont pas suffisamment approchés pour les besoins de la pratique toutes les fois qu'on ne pourra pas recourir à l'emploi d'un déversoir. Les résultats obtenus dans les expériences suivantes semblent répondre par l'affirmative.

Ces essais ont été faits avec toutes les précautions voulues, à la fois et en mêne temps, avec un moulinet immergé dans le canal d'amenée d'une usine hydraulique et sur un déversoir installé sur le canal de fuite de la même dérivation. Les opérateurs étaient MM. Magenties, Bellet et le soussigné.

Le canal de tuite, creusé dans le sous-sol de l'usine de MM. Jarrosson, à Bourg-Argental (Loire), était difficilement accessible, aussi les moyens ordinairement employés pour la mesure des hauteurs d'eau sur le déversoir auraient été peu commodes à effectuer et auraient pu, de ce fait, manquer un peu de précision. En outre, si une mesure sur un déversoir peut se faire en quelques minutes, il n'en est pas tout à fait de même avec un moulinet qui exige un certain temps et, comme il importait de s'assurer que la quantité d'eau absorbée par la turbine restait bien constante pendant toute la durée de la mesure au moulinet, il aurait fallu faire toute une série de mesures sur le déversoir. Afin d'éviter ces mesures successives, qui auraient exigé la présence permanente d'un opérateur supplémentaire, nous avons employé un hydromètre enregistreur de J. RichARD, de Paris; la lecture du diagramme inscrit par cet appareil a permis de se rendre compte à chaque instant de la hauteur de l'eau sur le déversoir. Nous nous sommes référés en cela à l'avis exprimé précédemment par $M$. Ribourt (*) que, grâce à la très grande précision avec laquelle cet hydromètre enregistre les plus minimes variations de niveau, il était tout indiqué pour observer le niveau de l'eau à l'amont du déversoir de jaugeage. On peut en outre tarer l'appareil sur place, avant ou après une mesure, en plongeant plus ou moins la cloche dans un tonneau.

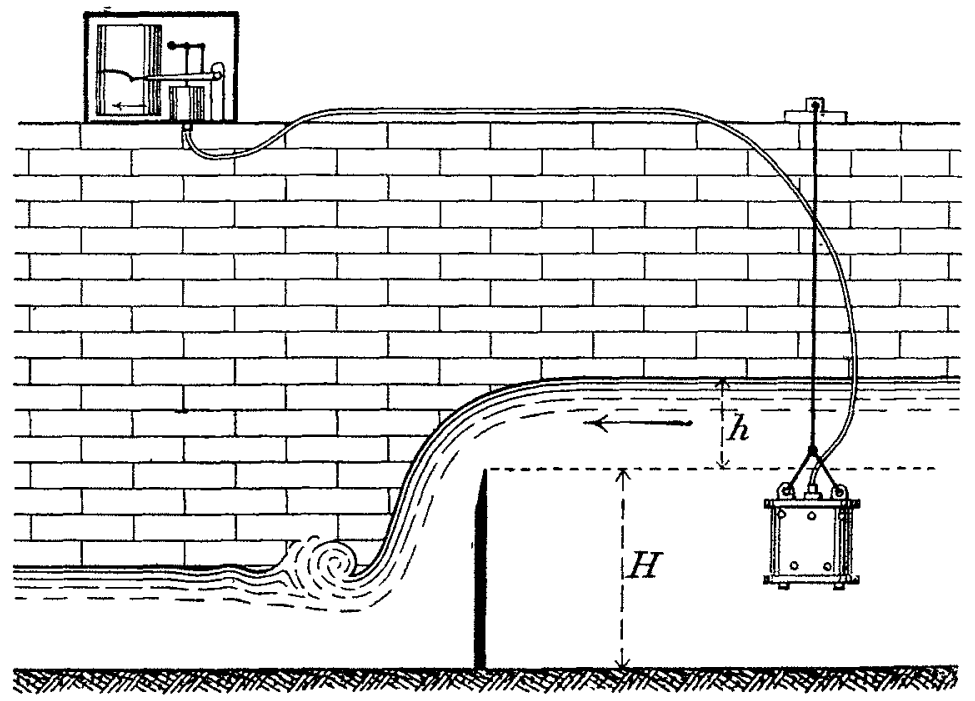

Fig. 1 .

La cloche en fonte de l'hydromettre était plongée dans l'eau du canal de fuite à environ $\mathrm{I}^{\mathrm{m}} 5 \mathrm{o}$ en avant du déversoir. Celui-ci était composé d'une planche surmontée d'une tôle taillée en biseau de manière à réaliser l'écoulement en mince paroi. Le déversoir ne tenait pas toute la largeur du canal; deux joues, ménagées de chaque côté, et taillées également en biseau, permettaient à l'air de pénétrer librement sous la nappe déversante, de manière à aroir una nappe libre et à éviter l'inconvénient des nappes plus ou moins déprimées. Un tuyau filiforme en cuivre reliait la poche flexible en caoutchouc, placée dans la cloche immergée, au cylindre du manomètre de l'enregistreur qui était posé à une certaine hauteur au-dessus de l'eau (voir fig. I). Pour mesurer la hauteur de la charge sur le déversoir, il suffisait de connaître, sur le diagramme enregistré, la valeur de l'ordonnée correspondant à une arrivée d'eau nulle et, pour cela, il a suffi d'arrêter un instant la turbine pendant l'heure du dîner du personnel, et de faire tourner un peu le cylindre de l'enregistreur pour lui faire tracer la droite correspondant au niveau de l'arête du déversoir.

Comme les vannes d'arrêt ne sont généralement pas étanches, les fuites, si l'on n'a pas vidé la chambre d'eau,

(*) Voir La Houlle Blanche, février 1905. 
se traduisent par une certaine lame d'eau sur l'arête du déversoir. Il pourrait être malaisé de déterminer exactement la cote de la crête de ce déversoir, si l'on n'a pas soin d'employer un artifice que nous croyons utile de signaler ici : il consiste simplement à munir le déversoir d'un petit orifice auxiliaire d'évacuation, obturé en temps ordinaire, un vulgaire robinet, par exemple, et qui permet, au moment du repérage, de laisser écouler l'eau provenant des fuites des vannes d'arrêt.

Voici quelles ont été les dimensions relevées pour ce déversoir : largeur du déversoir, $l={ }_{1} \mathrm{~m}_{105}$; largeur du canal de fuite, $L={ }_{{ }_{1}}{ }^{\mathrm{I}} 70$; hauteur de l'arête au-dessus du seuil, $H=0^{m} 38$; hauteur de la charge sur le déversnir, $h=\mathrm{o}_{2} \mathrm{rg}$.

Pendant que l'hydromètre, abandonné à lui-même, sans aucune surveillance spéciale, fonctionnait sur le canal de fuite, un jaugeage fut exécuté sur le canal d'amenée au moyen d'un moulinet de Woltmann, modèle nouveau construit par Orr, de Munich. Un plateau en bois fut jeté en travers du canal, en BD de la figure 4 ; le moulinet fut fixé à une baguette et immergé dans le canal suivant 18 positions successives représentées sur la figure 2. On appréciait le nombre de tours par la mesure du temps écoulé entre deux sonneries successives et, en se reportant à la courbe de tarage de l'appareil, on avait pour chaque position la vitesse moyenne correspondante.

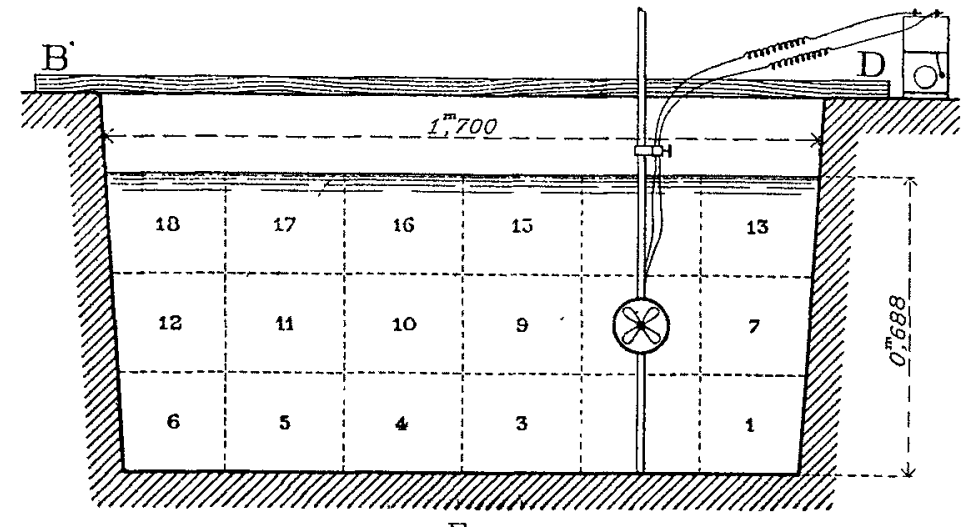

Fig. 2.

Il nous a paru que, pour les faibles vitesses, il était préférable d'employer, au lieu d'une sonnerie, un galvanomètre, ou un petit voltmètre, comme nous le fìmes en d'autres occasions, et d'observer la déviation de l'aiguille sous l'action de l'extra-courant de rupture.

\begin{tabular}{|c|c|c|c|c|c|c|}
\hline Sections $n^{0 s}$. & 1 & 2 & 3 & 4 & 5 & 6 \\
\hline Vitesses en centimètres. & 20.8 & 23,2 & 22,9 & 21,2 & 19,5 & 17,2 \\
\hline Sections $n^{0 s}$. & 7 & 8 & 9 & 10 & 11 & 12 \\
\hline Vitesses en centimètres. & 21,5 & 24,0 & 23,0 & 21,5 & 20,1 & 18,0 \\
\hline Sections $n^{o s}$. & 13 & 14 & 15 & 16 & 17 & 18 \\
\hline Vitesses en centimètres. & 23,9 & 22,0 & 20,1 & 18,5 & 16,0 & 15,5 \\
\hline
\end{tabular}

Les résultats obtenus sont consignés dans le tableau cidessus, et ils sont représentés graphiquement par les courbes de la figure 3. Les ordonnées de ces courbes sont comptées à partir d'un axe ayant pour ordonnée celle qui correspond à $V=\mathrm{om}_{\mathrm{I}} \dot{0}$; elles sont par suite proportionnelles à $V-0,10$.

Les vitesses maxima observées ont été de $\mathrm{om}_{24}$ o au milieu de la hauteur, de $\mathrm{o}_{2} \mathrm{~m}_{2}$ au fond et $\mathrm{de}^{\mathrm{m}_{2}} 3 \mathrm{~g}$ à la surface Il est à remarquer que, pour une même section horizontale, les vitesses semblent décroître au milieu d'une façon linéaire, et que l'influence des parois se fait brusquement sentir. On voit que le maximum, au lieu de se trouver au milieu du canal, se trouve vers l'un des bords. Cela tient à une double cause. D'abord à la disposition du canal dont la figure 4 représente une partie du plan. Par suite du coude rétréci en $\mathrm{AC}$ et de la direction tangentielle de la paroi C D, le courant était naturellement plus fort en $D$ qu'en B. L'état des lieux n'avait pas permis de choisir un autre emplacement, le canal longeant le rocher à pic sur

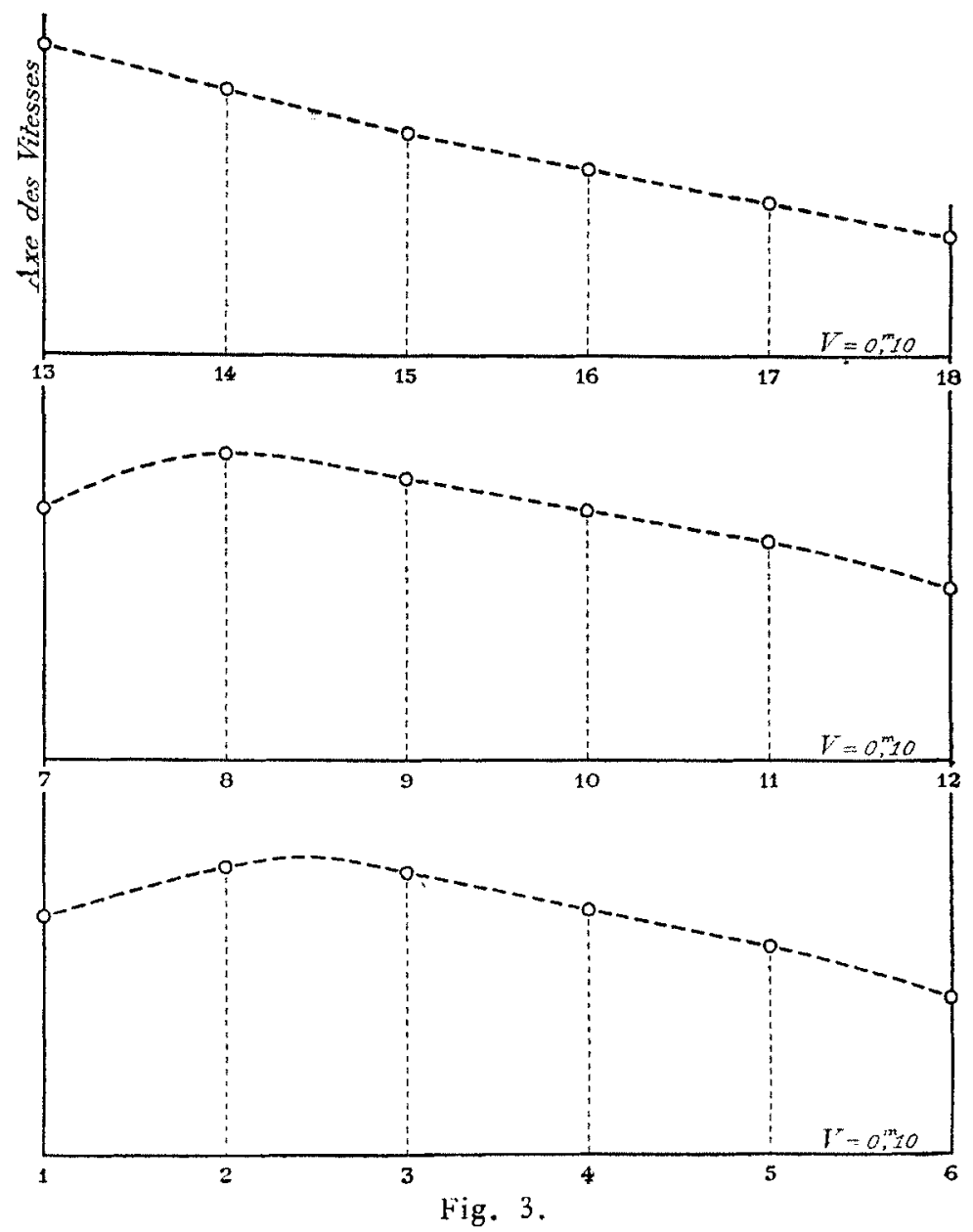

presque tout son parcours. En outre, le vent soufflait obliquement au canal et en sens contraire du mouvement de l'eau, amortissant ainsi la vitesse superficielle de l'eau sur le côté A B.

La largeur du canal, au niveau de l'eau, était de $1^{\mathrm{m}} 7^{\circ}$ et la profondeur de l'eau de om688. Le fond du canal est lisse et horizontal suivant sa direction transversale, sa pente lon. gitudinale est de $5 \mathrm{~m} / \mathrm{m}$ par mètre. Les côtés latéraux, inclinés à 5 pour roo, sont en moellons simplement rejointoyés. Les maçonneries dépassaient le plan d'eau de 20 centimètres, abritant ainsi l'eau du côté C D.

En faisant la somme des produits des vitesses par les sections élémentaires correspondantes, on trouve un débit de 236 litres. 
A l'extrémité du canal de fuite, et tout à fait à l'entrée de la chambre de mise en charge de la conduite forcée, se troụvait une vanne de purge. Cette vanne n'était pas absolument étanche et donnait lieu à quelques fuites dont le débit était très sensiblement de un litre à là seconde, de sorte que la quantité d'èau absorbée par la turbine était, d'après le moulinet, de 235 litres.

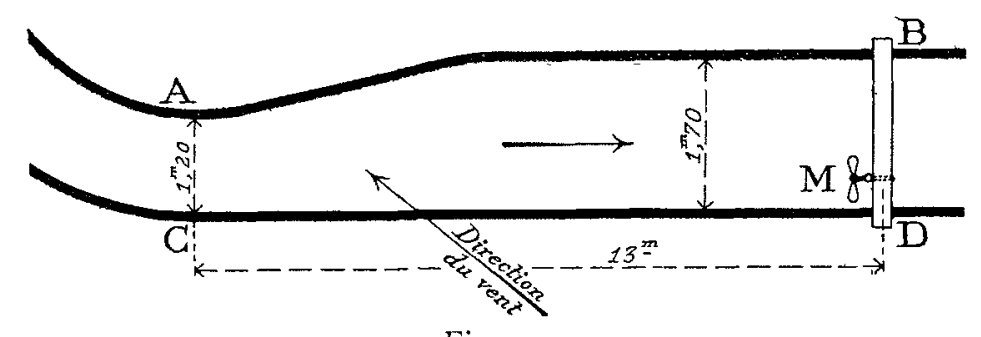

Fig. 4.

Pendant le temps, assez long, nécesssaire à la mesure au moulinet, la puissance de la turbine, qui fonctionnait sous une chute de 20 mètres, est restée très sensiblement constante et il en a par suite été de même pour le débit. En effet, deux tiers de l'énergie mécanique produite par la turbine étaient absorbés par un moulinage de soie et l'autre tiers par un atelier de tissage. Or, l'on sait que la puissance requ se par un moulinage est, en marche normale, rigoureusement constante. Dans l'atelier de tissage il n'en est évidemment pas de même, car, à tout instant, un métier s'arrète pour le raccommodage d'un fil cassé, mais, en général, quand un métier s'arrête, un autre se remet en marche; de sorte que la variation de puissance de l'atelier peut être estimée égale à celle d'un métier, soit un quart de cheval et, finalement, la variation du débit, à $r$ ou 2 litres at maximum. C'est-à-dire que cette variation de débit est insignifiante et de l'ordre de grandeur des erreurs que l'on peut commettre dans la mesure au déversoir ou au moulinet. D'ailleurs l'hydromètre a enregistré une ligne droite n'accusant que des oscillations microscopiques.

La formule générale qui donne le débit d'un déversoir est, comme on le sait :

$$
Q=m l h \sqrt{2 g h l}
$$

$m$ étant un coefficient pratique qui varie avec les dimensions du déversoir.

En Suisse et en Allemagne on emploie beaucoup la formule de M. Braschmann:

$$
m_{1}^{\prime}=0,3838+0,0386 \frac{l}{L}+\frac{0,00052}{h}
$$

qu1 donne ici : $m_{1}^{\prime}=0,3862+0,0386 \frac{l}{L}=0,4227$.

Or, s'll n'y avait pas eu contraction latérale (dans le cas de $l=L$ ), on aurait eu $m_{1}=0,4248$; il en résulte que le rapport de $m_{1}^{\prime}$ à $m_{1}$ est de 0,995 .

En France, on se sert à peu près exclusivement des formules Boileau ou Bazin, mais surtout de la dernière :

$$
\begin{array}{ll}
m_{2}=0,17 \frac{h+H}{\sqrt{(H+h)^{2}-h^{2}}} & \text { (Boileau) } \\
m_{3}=n_{i}\left[1+0,55\left(\frac{h}{h+H}\right)^{2}\right] & \text { (Bazin) } \\
\text { avec } \mu=0,421 \quad 0,417 & 0,414 \\
\text { pour } h=0,20 \quad 0,30 & 0,40
\end{array}
$$

Ces deux formules, établies pour les cas où il n'y a pas de contraction latéralc, tiennent compte toutes deux de la vitesse d'arrivée de l'eau sur le déversoir, vitesse qui est d'autant plus faible que la hauteur $H$ est plus grände. Or, plus la vitesse d'arrivée est faible, plus le débit doit être petit pour une même valeur de $h$; c'est bien là, d'ailleurs, ce qu'indiquent les deux formules qui donnent très sensiblement les mêmes résultats.

La formule Boileau donne ici $m_{2}=0,448$ : Pour la formule Bazin, en prenant $\mu=0,420$ qui semble bien être la valeur correspondante pour $h=0,22$, on trouve $m_{3}=0,45 \mathrm{r}$.

Comme la largeur du déversoir est plus petite que celle du canal de fuite, il faut corriger un peu les coefficients précédents. La diminution de largeur du déversoir a pour premier effet de diminuer un peu la vitesse darrivée de l'eau, mais, si cette vitesse est moins grande, l'influence des parois du canal se fait moins sentir; pour un déversoir sans contraction latérale la vitesse doit être plus faible sur les bords qu'au milieu, de sorte qu'il y a la un peu compensation. Mais l'effet principal du rétrécissement du déversoir provient de la contraction latérale et l'influence de cette contraction est d'autant plus petite que le déversoir est plus large. Certains hydrauliciens ont estimé que dans le cas de déversoirs de grande largeur et pour $l=0,9 \circ L$, on devait avoir $m^{\prime}=0,99 \mathrm{~m}$; comme ici $l=0,95 L$ environ ori devrait donc avoir $m^{\prime}=0,995 \mathrm{~m}$. Ce coefficient de correction est exactement le même que celui de la formule Braschmann, aussi l'adopterons-nous comme coefficient de correction de la formule Bazin qui donne alors $m_{3}^{\prime}=0,4 s_{7}$.

Ce coefficient de la formule (Bazin $m_{3}^{\prime}=0,449$ ) differe notablement de celui de la formule Braschmann $\left(m_{1}^{\prime}=0,+23\right)$, bien que nous ayons employé le même coefficient de contraction latérale. Cela vient très probablement de ce que cette dernière formule ne tient pas compte de la hauteur $H$ et, par suite, de la vitesse d'arrivée de l'eau. La formule Braschmann ne convient, par suite, que pour des valeurs de $H$ très grandes ; c'est ainsi que, pour $H=1^{\text {m }} 5 c$ et $h=0,22$ elle donne, sans contraction latérale, la même valeur que la formule Bazin, $m^{\prime}=0 ; 4^{2} 4$.

Pour calculer le débit sur le déversoir, nous nous servirons de la formule Bazin, qui nous paraît le mieux tenir compte des diverses dimensions. En portant $m_{3}=0,4487$ dans la formule générale, on trouve un débit de 225 litres.

L'écart entre les deux jaugeages est donc de 10 litres, soit 4,35 pour roo sur la moyenne des deux mesures. Cet écart de 4,35 pour 100 est la somme de quatre quantités :

$\mathrm{I}^{\circ}$ L'erreur relative commise dans la mesure des dimen. sions du déversoir;

$2^{\text {o }}$ L'erreur relative due à l'emploi d'une formule qui, si approchée qu'elle soit de la réalité, n'en -est pas moins une formule empirique ;

3o L'erreur relative commise dans la mesure des dimensions sur le canal d'amenée et, enfin:

$4^{\circ}$ L'erreur relative provenant du tarage du moulinet.

Dans son étude sur les moulinets, M. Rateav a montré que cet appareil devait donner en pratique des résultats légèrement trop forts. Cela vient de ce que les moulinets sont généralements tarés. par déplacement de l'appareil en 
eau tranquille et que, dans cette opération, les divers filets liquides frappent tous les aubes avec la même vitesse, tandis qu'en pratique il n'en est pas tout à fait ainsi; la non égalité de vitesses des filets liquides frappant les aubes conduit à une vitesse du moulinet plus grande que ne l'aurait donné une vitesse uniforme des filets liquides égale à leur vitesse moyenne. En plus de ceci, il faut tenir compte de l'erreur relative qui a été commise dans la mesure des paramètres effectuée pendant le tarage, de sorte que l'on peut estimer que la $4^{\bullet}$ erreur relative est du même ordre de grandeur que les trois autres.

En admetlant donc le cas le plus favorable, c'est-à-dire celui ou les mesures sur le déversoir pêcheraient par défaut et celles sur le canal par excès, on voit que l'erreur relative propre à chacun des quatre cas ne serait plus que que de I pour 100 , chiffre bien faible si l'on remarque que ces mesures n'ont point été exécutées dans un laboratoire, ni après des préparatifs faits spécialement dans ce but, mais au cours de la marche normale - industrielle d'une usine hydraulique, et dans le simple but de rechercher l'ordre de grandeur de l'écart possible entre les deux procédés dans certaines conditions déterminées.

L'appareil enregistreur Richard était du type couIdnt, ordinairement employé pour le relevé des diagrammes des distributions d'eau. Le cylindre enrégistreur tournait à raison de 1 tour par $2+$ heures et la hauteur du papier était de 10 centimètres pour une variation maximal du niveau de

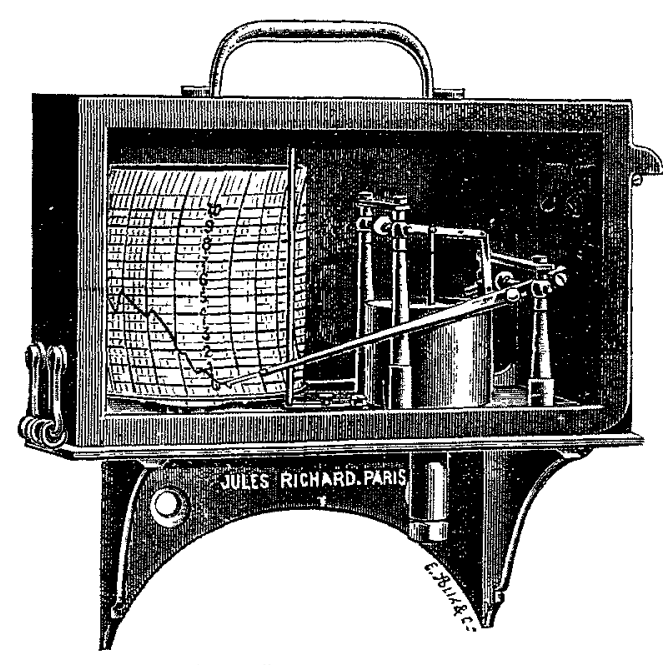

Fig. 5. - Enı egistreur Richard. l'eau de I mètre, de sorte qu'il fallait apprécier le dixième de millimetre, ce qui a pu se faire, grâce à la netteté du diagramme enregistré. Toutefois, il sera préférable d'employer un cylindre grand modèle de 15 centimètres pour une variation maxima de 50 centimètres d'eau, de manière à n'avoir plus à apprécier que le quart de millimètre.

Ces essais n'ont point été faits dans le but d'en tirer une conclusion pour les lecteurs de La Houille Blanche; comme ils ont été exécutés pour notre seule édification personnelle nous gardons pour nous les réflexions qu'ils nous suggèrent, et nous ne les relatons ici qu'à titre documentaire. Nous en dégagerons seulement ce conseil que pour une mesure sur un déversoir, on pourra employer avec avantage un hydromètre enrégistreur et cet avis que, dans le cas d'un cours d'eau canalisé, à écoulement régulier, les mesures au moulinet peuvent être considérées comme exactes à 5 pour 100 près.

$$
\text { E.-F. Còtr. }
$$

\section{LE MOIS HYDRO-ÉLECTRIQUE}

\section{ACADÉMIE DES SCIENCES}

\section{GEOLOGIE ET HYDROLOGIE}

Sur une nouvelle exploration du gouffre du Trou-de-Souci (Côte dOr). - Note de M. E. A. Martel, séance du 17 juillet.

A $57 \mathrm{~m}$. de profondeur l'eau itait stagnante; en mars-avril on lavalt entendue couler avec force; cet écoulement mtermittent se fait àu sud-ouest au nord-est (orientation de la courbe extérieure) el peut s'élever de $6 \mathrm{~m}$. à $10 \mathrm{~m}$. datis le fond du gouffre. En amont, nous avons reconnu. avec deux canots de toile, un lac souterrain de $50 \mathrm{~m}$. de longueur, 3 à $12 \mathrm{~m}$. de largeur, I $\mathrm{m} 50$ à $4^{\mathrm{m}} 40$ de profondeur, dans une série de diaclases (hautes de 10 à $20 \mathrm{~m}$.) parallèles entre elles et que l'eau à mises en communication par leur base; toute crue de $2 \mathrm{~m}$. a $3 \mathrm{~m}$. immerge les pointes de leurs cloisons rocheuses séparatives et les amorces en vases cummunicants (zdem à Marbl Arch en Irlande). Une yotite mouillante ainsi immergée de oul ro nous a arrêtés. Les concrétions manquent, nayant pas le temps de se déposer sur les parois entre deux crues successives. mais une abondante précipitation calcique transparait au fond de l'eau saturée de carbonate de chaux. Il n'y a que de faibles dépôts d'argile, presque toute entraìnée par les flux; l'un d'eux est percé, en écummoire, de petits entonnorrs coniques creusés par les suintements des hautes voûtes. La température de l'eau est de $1^{\circ}$, celle de l'arr de $9^{\circ} 5$, à cause du courant d'air tombant du gouffre et qui active l'évaporation.

En aval, les diaclases, hautes de $20 \mathrm{~m}$. à $30 \mathrm{~m}$. et en partie à sec. forment un labyrinthe et s'anastomosent atx hautes caux; toutes se rétricissent à oll $_{40}$ où à om 60 (avec $4 \mathrm{~m}$. de profondeur d'eau) et sont intranchissables a pied comme en bateau. (Idem aux avens de la Cêpe (Drôme), de Sauve (Gard), ete., mars leur partie moyenne est renflée en fuseau (Bramabıau, Padirrac, Bétharram, etc.); le gouffre lu1-même est la principale de ces cassures, propagée jusqu'au sol, et ouverte en abìme par la capture de l'ancien écoulementde la courbe. Une autre crevasse volsine s'élève aussı jusqu'à la surface, mais son orifice y est bouché.

Les indıces de corrosion chimıque et de mise en liberté de l'argile du calcaure sont très nets. A l'aval, l'érosion mécanique a accumulé un dépôt de milliers de galets calcaires roulés, polis, sphériques où ovoides (de $\mathrm{O}_{\mathrm{O} 2}$ à $\mathrm{o}^{\mathrm{m}_{1}} 5$ de diamètre) pareils à ceux trouvés, en I 883 . lors de l'éhoulement du tunnel du Crédo (Aın), tte. ; ils témoignent de courbillonnemennts intenses et d'effets dynamiques très puissants, majs surtout dans les boyaux inférieurs ou vases communiquanı, rraisemblablement sinueux, où les crues font chasse en condutes forcées. Dans les galeries libres à l'étrage, la propagation des eaux Joit ètre plus calme, car nous avons retrouvé en aval, à fleur du sol, et intacts, une corde (laissée par nous le 24 octobre 1904 sur le bord du gouffre) et les ossements (mâchoire. etc). d'un suicidé dc mars 1885 ; le tout a été tranquillement fotté à si petite distance que, selon toute présomption, le labyrinthe n'aboutıt qu'àdes voûtes mouiliantes comme le lac d'amont.

En résumé, le Trou-de-Souci synthetıse d'une façon toute particulierement remarquable les lois principales de l'hydrolngie des calcaires savoir :

$1^{\circ}$ Préexistance des fissures du sol (joints et diaclases).

20 Capture, parles cassures recoupant la surface des cours d'ean extérieurs (sans doute dès le tertiaire).

30 Agrandissement de cescrevasses capturantes en pertes et abìmes de haut en bas en général; exceptionnellement de bas en haut, par effrondement postérieur.

$f^{\circ}$ Dessèchement progressif des vallées par les caprures (achevé au Souci, dans les Causses, etc., en cours d'exécution dans le Jura, la Charente, sur le haut Danube, en Belgique, etc.).

$5^{\circ}$ Dilatation souterraine des diaclases en citernes distinctes, parfois raccordées en assez longues rivières intérieures.

$6^{\circ}$ Triple action de l'érosion, de la corrosion et de la pression hydrostatique. Décalcification contemporaine très notable.

70 Décadence de l'écoulement souterrain, les parties basses des cavités étant moins dilatées que les patties moyennes.

80 Circulation souterraine non pas en nappes, mais en réseau de fissures très solvent anastomosée.

go Intermittence (parfois jusqu'à la stagnation complète) et, en tout cas, irrégularité extrême de cette circulation, selon l'abondance Jes pluies et des infiltrations du dehors (explqquant les variations des résurgences et leur temporaire contamination accidentelle).

100 Remplissage des 'issures-résersolrs en hauteur, bien plus qu'en largeur, avec des mises en charges qu'on a vues supérieures a 10 atmosphères dans certains gouffres du Karst.

I $1^{\circ}$ Amorçages et jeu de siphons (d'aqueducs) souterrans (vases communiquants) lors des crues souter raines.

$12^{\circ}$ Propagation tantót calme, tantót violente, des eaux sonterarines selon la forme et le diamètre de leurs conduites. 